Recent Insights into the Physics of the Sun and Heliosphere:

Highlights from SOHO and Other Space Missions

IAU Symposium, Vol. 203, 2001

P. Brekke, B. Fleck, and J. B. Gurman eds.

\title{
Spectroscopic Signatures of a Flare Observed by SUMER Onboard SOHO
}

\author{
I. E. Dammasch, W. Curdt \\ Max-Planck-Institut für Aeronomie, Katlenburg-Lindau, Germany \\ B. Kliem \\ Astrophysikalisches Institut, Potsdam, Germany \\ B. N. Dwivedi \\ Banaras Hindu University, Varanasi, India
}

K. Wilhelm

Max-Planck-Institut für Aeronomie, Katlenburg-Lindau, Germany

\begin{abstract}
We report on EUV observations of a solar limb flare obtained by the SUMER spectrometer. A time series was taken with fixed slit position and several spectral windows that covered a wide temperature range $\left(10^{4}-10^{7} \mathrm{~K}\right)$, preceded and followed by contextual raster scans in a He I line. During the time series, a C4.6 flare occurred in the region, also imaged in the EUV by SOHO/EIT and in soft X rays by YOHKOH/SXT. The temporal evolution seen in the SUMER spectra reveals a close spatial relationship and a correlated dynamical behaviour of the hot $\left(T \approx 10^{7} \mathrm{~K}\right)$ and cool $\left(T \approx 10^{4} \mathrm{~K}\right)$ material, which are difficult to reconcile with the notion (based on the Kopp-Pneuman flare model) that cool loops form at a lower height than the hot flare loops.
\end{abstract}

During a SOHO campaign of the flare watch series, the SUMER spectrometer (Wilhelm et al. 1995) observed a flare (GOES C4.6) above the north-east limb in NOAA AR 8758 on 6 November 1999 after $\approx 06: 30$ UT. The building block of the SUMER programme consisted of 25 exposures of the $300 \times 1$ arcsec slit on detector A of $120 \mathrm{~s}$ each, simultaneously taken in eight spectral windows, followed by transmission of three full-detector spectra (16 min). This block was repeated 8 times at a fixed slit position above the limb, and the whole sequence was preceded and followed by a contextual raster scan in the He I $584 \AA$ line formed at $T \approx 2 \times 10^{4} \mathrm{~K}$. Fig. 1 shows the images built from the two raster scans and the intensity data of the time series in a representative cool line (C II $1335.7 \AA$ formed at $T \approx 2 \times 10^{4} \mathrm{~K}$ ) and a representative hot line (Fe XXI $1354.1 \AA$ formed at $T \approx 10^{7} \mathrm{~K}$ ). Lines formed at similar temperatures behaved similarly.

The two raster scans and the available EIT images exhibit a slow rise of the main loop systems during the observation (a small part of which is due to 

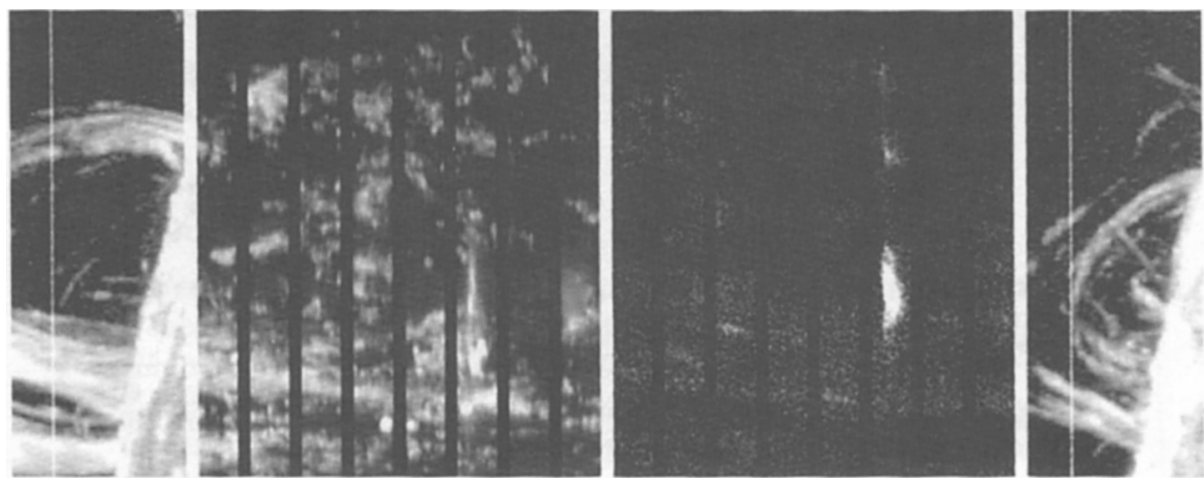

Figure 1. He I images of the active region obtained from the initial (00:33-47 UT) and final (09:41-55 UT) SUMER raster scans of the area $1028-926^{\prime \prime} \mathrm{E} \times 100-400^{\prime \prime} \mathrm{N}$ (left and right panels) and line-integrated intensity time series at fixed slit position in the C II and Fe XXI lines (2nd and 3rd panel from left, respectively). The slit position at $1012^{\prime \prime} \mathrm{E}$ is indicated in the context images. Time is running from left to right (00:48-09:25 UT).

solar rotation). The time series data clearly display the flare enhancement of the Fe XXI line during $\approx 06: 30-07: 10$ UT. Enhancements in C II are seen to occur intermittently throughout the whole time series. Fig. 1 suggests that this reflects the passage of a rising highly complex system of cool loops across the SUMER slit. We focus here on the C II enhancement that is spatially and temporally most closely related to the Fe XXI enhancement (leaving further aspects for a subsequent, more detailed investigation). It shows a particularly impulsive rise and is almost certainly related to the flare. Lines formed at typical coronal temperatures (Fe XII 1349.4 $\AA$, Si IX $676.5 \AA$, Al X $670.0 \AA$, Ar XII $670.3 \AA$ ) were monitored, but did not show any obvious enhancement related to the flare. The latter fact may be due to a small filling factor of the plasma involved in the flare process relative to the column density of the surrounding coronal material at similar temperatures.

The enhancements of C II and Fe XXI occurred both in the range $\approx 200$ $240^{\prime \prime}$ (with their maxima offset along the slit by $\approx 10^{\prime \prime}$ ). The temporal evolution of the EUV flux integrated over this range is shown in Fig. 2(a), along with the GOES soft X-ray light curve. The onset of the Fe XXI enhancement is delayed by $Z 2$ min with respect to the strongest rise of the soft $X$ rays (i.e., to the impulsive flare phase); this indicates that the SUMER slit did not happen to sit exactly on the flare site. The nearly simultaneous onset of the C II and Fe XXI enhancements at a time after the impulsive phase strongly suggests that the cool and hot plasmas did not originate at different places (e.g., downward draining of cool plasma and simultaneous rise of "evaporated" hot plasma); the magnetic structures containing these plasmas have probably moved jointly toward the slit position. An EIT $195 \AA$ image at 06:35 UT also shows a brightening slightly below the SUMER slit close to the C II and Fe XXI enhancements, which was 



Figure 2. (a) Line-integrated intensity and (b) line shifts, summed up along a section (200-240" N) of the slit in three EUV lines during the SUMER time series. The GOES 0.5-4 $\AA$ time profile is included.

absent in the preceding image at 06:26 UT and is hard to distinguish from other structures in the subsequent image at 06:52 UT.

The onset of the C II and Fe XXI enhancements occurred nearly simultaneously, but the peak of the C II enhancement preceded the peak of the Fe XXI enhancement, and the C II enhancement decreased earlier than the Fe XXI enhancement! This sequence is not consistent with the standard notion of the Kopp-Pneuman flare model that cool loops (in particular post flare loops) form below the hot flare loops (by radiative and conductive cooling of those dense loops) with temporally increasing formation height. Although the cool plasma in this flare could be observed only after the impulsive phase, we conclude that it was more closely related to the hot plasma than the standard theoretical model suggests.

This conclusion is supported by the observed line shifts and line broadenings. The line shifts of the C II and Fe XXI lines appeared to be oscillatory, and they showed a high degree of (anti-) correlation [Fig. 2(b)]. Both lines were substantially broadened only during the flare-related enhancement.

An interpretation of the hot plasma flows as chromospheric evaporation is unlikely, due to the reversal of the flow direction. Moreover, the spectral signatures of evaporation reported in the literature have in general declined by the time of peak soft X-ray flux. It is more probable that we see a dynamical loop behaviour similar to the observations by Kjeldseth-Moe and Brekke (1998), but related to the flare disturbance.

Acknowledgments. The SUMER project is financially supported by DLR, CNES, NASA, and the ESA PRODEX programme (Swiss contribution).

\section{References}

Kjeldseth-Moe O., Brekke P. 1998, Solar Phys., 182, 73

Wilhelm K., et al. 1995, Solar Phys., 162, 189 